\title{
Prison et liberté d'après Latifa Al-Zayyat, Malika Oufkir et Tahar Ben Jelloun
}

\section{Ayman Amin El Ghandour*}

aymaneamen@yahoo.fr

\section{Résumé}

Cette étude met l'accent sur la littérature carcérale à travers Perquisition de Latifa Al-Zayyat, La prisonnière de Malika Oufkir et Cette aveuglante absence de lumière de Tahar Ben Jelloun. Latifa et Malika ont présenté deux récits autobiographiques alors que Ben Jelloun a mêlé la réalité à la fiction dans son récit, dédié à Aziz Binebine. Les trois romanciers ont bien décrit la violence politique, adoptée par l'État face à ses opposants, dénonçant le régime despotique dans leurs pays. Ils ont montré les mesures répressives, exprimant la souffrance des détenus dans des prisons inhumaines: Bir Jdid, Kanater et Tazmamart. Ceux-ci ont réussi à dompter leurs émotions afin de résister aux conditions pénibles pendant leur détention. Ceci parait évident à travers le personnage de Salim qui s'est consacré au soufisme.

Les mots clés: Littérature carcérale, prison, liberté, Tazmamart, autobiographie.

* Professeur adjoint à la Faculté de Pédagogie de Tanta

(Prison et liberté d'après ...) Dr. Ayman El Ghandour 
"De nombreux écrivains ont été incarcérés, leur séjour en prison les a inspirés; les femmes ont contribué, avec leur collègues masculins, à créer un genre littéraire né de cette expérience, qu'on a appelé en Égypte la littérature des prisons". (Tadié, 1996, p. 71)

Prison et liberté sont deux mots étroitement liés si bien qu'il est difficile de séparer l'un de l'autre. Les détenus qui considèrent la prison comme une tombe, ne cessent de penser à la liberté, symbole de la vie. Ils y mènent une vie pénible, privés de tout. Leur détention est "une suspension de la vie sociale" (Bazié et Ferrer, 2015, p. 47). Le thème de la prison est non seulement présent dans les écrits littéraires, mais il a donné naissance à la littérature carcérale. Celle-ci s'intéresse à mettre en relief les détenus, leur expérience, la violence politique, l'autorité despotique et la privation des droits de l'homme. Cette littérature a connu un succès remarquable en Égypte et au Maroc, présentant tant d'ouvrages dont nous citons à titre d'exemple: Mémoires de la prison des femmes (1984) de Nawal El Saadawi, Première année en prison (1989) de Mostapha Amin, Bareaux (2008) de Mohamed Al Bossaty, Tazmamart cellule 10 (2001) d'Ahmed Marzouki, y compris le corpus de notre étude: Perquisition de Latifa Al-Zayyat (1992/1996), La prisonnière (1999) de Malika Oufkir et Cette aveuglante absence de lumière (2001) de Tahar Ben Jelloun.

L'originalité de cette étude réside dans le choix de notre corpus où Latifa Al-Zayyat et Malika Oufkir ont présenté leur expérience carcérale, alors que Ben Jelloun a abordé celle d'Aziz Binebine, un des détenus de Tazmamart. Cette authenticité est renforcée par la valeur de nos trois écrivains qui ont refusé de s'incliner devant des régimes despotiques et qui cherchaient sans cesse leur liberté et celle des autres. Appartenant à la gauche, ils

(Prison et liberté d'après ...) Dr. Ayman El Ghandour 
ont essayé de déceler l'injustice, dénoncer l'atrocité, dire l'indicible et présenter aux lecteurs la vérité telle qu'elle était.

La littérature carcérale est "un acte qui traverse les genres" (Coquio, 2015, p. 181); autrement dit, elle est un carrefour où se rencontrent l'autobiographie et le récit socio-historique. Ceci nous pousse à adopter plusieurs approches en étudiant les trois récits de notre corpus: la critique historique qui vise à montrer la crédibilité des écrits-témoignages et à comparer les documents officiels au texte littéraire; l'approche comparative, basée "sur une poétique comparée des formes, des genres ou des thèmes" (Souiller et Troubetzkoy, 1997, p. 2); l'approche autobiographique ayant pour but d'étudier les motivations qui poussent un écrivain à raconter son expérience. Dans cette optique, nous sommes guidé par Le pacte autobiographique de Philippe Lejeune et les écrits de Franck Evrard qui confirme que "L'écriture autobiographique peut avoir aussi une fonction testimoniale" (2006, p. 79). En tant que témoin, l'autobiographe raconte sa vie et celles des autres. Aussi proposons-nous des théories concernant la violence et les émotions, deux thèmes présents dans les prisons, comptant sur Les émotions de Jacques Regard, Les formes de la violence de Xavier Crettiez, Sémiotique des passions de Greimas et Fontanille. Tout cela nous aide à faire une lecture analytique et globale, à montrer les points de ressemblance et de différence. Nous essaierons de répondre à ces questions qui constituent la problématique de notre étude: Quelle est la valeur littéraire de nos trois écrivains? A quel point ont-ils exprimé leur expérience carcérale ou celle des autres dans les trois récits de notre corpus? Est-ce qu'ils ont présenté avec crédibilité le contexte historique de leurs récits? Pourquoi ont-ils écrits ces témoignages? Comment ont-ils contribué à défendre la liberté des opprimés? Comment les personnages ont-ils dompté leurs émotions? Quelles sont les

(Prison et liberté d'après ...) Dr. Ayman El Ghandour 
convergences et les divergences à travers la technique romanesque, utilisée par les trois écrivains?

A priori, il nous semble nécessaire de présenter les trois écrivains qui sont à la base de notre étude. Commençons tout d'abord avec Latifa Al-Zayyat. Elle est une romancière égyptienne, née en 1923. Après des changements de domicile, elle s'est installée au Caire où elle a achevé ses études supérieures. Étudiante, sa vie est marquée par son engagement politique; elle se trouvait parmi les dirigeants du Comité des étudiants. Elle a obtenu le doctorat en littérature anglaise. Son activisme politique et ses études ont enrichi sa production littéraire. Amina Rachid désigne que Latifa Al-Zayyat "s'impose dès les années 60 du siècle passé comme chef de file de l'explosion d'une riche écriture féminine dans l'ensemble du monde arabe" (2020, p. 107). C'est ce qu'affirme Christophe Ayad, annonçant que notre romancière "est la première écrivaine égyptienne traduite en français, à l'exception de l'égérie surréaliste Camille Joyce Mansour" (1996). Sa carrière littéraire est couronnée par le prix Naguib Mahfouz pour le roman qu'elle a obtenu dans sa première édition. Auprès de sa carrière de professeure à l'université où elle considérait l'enseignement comme un contact avec les étudiants, Latifa s'avère essentiellement une combattante permanente, à la recherche de la libération féminine et celle de son peuple face aux Anglais. Il en résulte qu'elle "a connu deux fois la prison d'abord en 1949, puis plus tard en 1981 encore" (Trutt, 2019).

Née en 1953, Malika est la fille de Mohamed Oufkir, ministre de l'intérieur au temps du roi marocain Hassan II. Agée de 5 ans, le roi Mohamed V l'a adoptée pour être compagne de la princesse Lalla Mina. Elle a passé son enfance et son adolescence au palais royal. En 1972, une surprise a bouleversé toute sa vie;

(Prison et liberté d'après ...) Dr. Ayman El Ghandour 
son père est exécuté après l'échec de son coup d'État. Comme la famille Oufkir a refusé l'aide royale, Hassan II a envoyé tous ses membres en prison secrète où ils sont restés 19 ans. Luc Levaillant dit à ce propos: "Malika Oufkir a passé les deux tiers de sa vie enfermée" (1999, le 22 février). Après sa fuite de la prison, elle s'est mariée avec un architecte français. Elle a commencé sa carrière littéraire avec La prisonnière (1999) qui raconte son expérience carcérale et L'étrangère (2008) où elle narre son retour à la liberté.

Quant à Tahar Ben Jelloun, il est né en 1944 à Fès; il a commencé à fréquenter l'école coranique. Agé de six ans, il a rejoint l'école franco-marocaine où il a étudié le français. Cinq ans après, il est parti à Tanger pour achever ses études secondaires. Après avoir obtenu son baccalauréat, il s'est installé à Rabat pour étudier la philosophie. "Mais à la suite des manifestations estudiantines de mars 1965, il est envoyé avec quatre-vingtquatorze autres étudiants dans un camp de redressement disciplinaire de l'armée" (Kadji, 2020, p. 14). Là, il a écrit "L'aube des dalles", son premier poème, publié plus tard dans Souffles, revue marocaine fondée par Abdellatif Laâbi en 1966. Après son retour à l'université en 1968, il a pu achever ses études, devenant successivement professeur de philosophie à Tétouan et à Casablanca. Lorsque le Maroc a arabisé la philosophie, Ben Jelloun est parti pour Paris où il s'est spécialisé en psychiatrie sociale; il a profité de cette carrière qui lui avait inspiré tant de thèmes et de personnages. Il a obtenu le doctorat en 1975; deux ans après, il a publié une partie de sa thèse, ayant pour titre $L a$ plus haute des solitudes. Il a commencé à rédiger des articles, publiés par Le Monde et d'autres journaux suédois, allemands et italiens. Ceci lui a permis d'être au courant de tout ce qui se passait sur le plan local et universel. Dans un entretien avec Judith

(Prison et liberté d'après ...) Dr. Ayman El Ghandour 
Wand, il dit: "Je travaille pour des journaux [...] C'est que je suis pas un écrivain de chambre, c'est-à-dire j'interviens dans l'actualité" (1993, p. 104).

Tahar Ben Jelloun nous a présenté une production littéraire tout à fait variée et riche: Hommes sous linceul de silence, recueil de poèmes publié en 1971, des romans dont nous mentionnons Harrouda (1973) et La réclusion solitaire (1976). Face au fanatisme et à l'intolérance, il a écrit Le racisme expliqué à ma fille (1998) et L'islam expliqué aux enfants (2001). Il a préféré écrire ses ouvrages en français parce qu'à ce temps-là, la langue française était imposée dans les écoles marocaines et les éditeurs les plus connus étaient français. Ces derniers avaient la capacité de présenter un écrivain à des millions de lecteurs. Grâce à ses écrits florissants, il a obtenu le prix Goncourt en 1987. De plus, il "siège depuis le 6 mai 2008 à l'Académie Goncourt" (Kadji, 2020, p. 16). Il n'a pas hésité à dénoncer les tares de la société marocaine, défendre la justice, la liberté et l'équité face au despotisme royal. Dans un entretien avec France 24, il a dit que le Maroc est "un pays où il y a des lois qui datent du Moyen-âge" (2019). Ceci paraît évident avec Cette aveuglante absence de lumière qui constitue le troisième volet de notre corpus.

Lorsqu'un détenu présente son expérience carcérale, son témoignage fait partie de la mémoire collective, inséparable de l'Histoire. Son écriture de soi est ainsi littéraire et historique où l'autobiographe narre ses impressions, dénonce les abus du régime despotique. C'est pourquoi, nous allons recourir au contexte politique au Maroc et en Égypte, abordé par les trois romans de notre corpus pour dévoiler la violence politique, exercée par l'État et ses institutions.

(Prison et liberté d'après ...) Dr. Ayman El Ghandour 


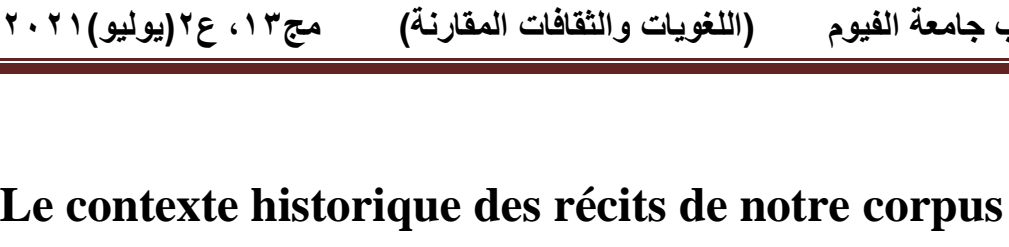

La deuxième moitié du siècle précédent est une époque riche en mutations politiques en Égypte et au Maroc. Elle se distingue par une atmosphère lugubre et une lutte permanente entre les mouvements de gauche et l'autorité despotique soit royale ou républicaine. Celle-ci ne tarde pas à faire construire des prisons entourées de hauts murs qui empêchent les détenus de voir le ciel ouvert. Dans cet espace d'étouffement, ces derniers sont victimes d'une agression physique et d'une violence politique. Celle-ci est légitime parce que c'est l'État qui en a le monopole. A ce propos, Crettiez dit: "C'est le droit qui donne force à la violence de l'État" (2008, p. 47). Dadoun affirme tout ce qui précède et décèle un lien étroit entre le pouvoir et la violence. "Le pouvoir affronte et utilise la violence, et celle-ci, en retour, exprime toujours une certaine forme de pouvoir" (1993, p. 58)

En Égypte, les officiers libres ont obligé le roi Farouk à abdiquer en 1952, mettant ainsi fin à la dynastie de Mohamed Ali. Après la nationalisation du canal de Suez, les Français ont essayé d'affaiblir le pouvoir de Gamal Abdel Nasser qui constituait une menace à l'État d'Israël. Après l'échec de l'agression tripartite, le monde arabe a tenu le président égyptien pour un leader. Il a réussi à expulser les Juifs, les Français et les Anglais. Mais à son temps, l'État hébreu s'est emparé du Sinaï en détruisant l'aviation égyptienne. Sur le plan intérieur, Nasser a adopté le socialisme au profit des petites gens. Cependant il a renforcé l'État policier, particulièrement après un attentat manqué contre lui. Nasser a de même lâché la bride à ses officiers pour pourchasser la confrérie des frères musulmans, ainsi que les communistes qu'il considérait agents du sionisme. Noha Khallaf désigne que "la plupart des intellectuels critiques de l'époque nassérienne ont été incarcérés"

(Prison et liberté d'après ...) Dr. Ayman El Ghandour 
(2015, p. 55). En prison, ils étaient privés de tout. Pendant la fouille, les gardes n'hésitent pas à déchirer leurs habits et à brûler leurs livres. L'eau dont ils buvaient, n'était pas potable et leur nourriture quotidienne était insuffisante. Le manque du service médical a fait d'eux une proie aux maladies infectieuses. "Au bagne de Tourah, on donna l'ordre de faire travailler les prisonniers politiques aux carrières où on les menait enchaînés sous un soleil brûlant (décembre 1954). Pour protester contre cette mesure inhumaine, les détenus commencèrent le 31 décembre une grève de la faim qui se poursuivit pendant 18 jours" (Éditions Esprit, 1956, p. 618, 619). Nous pouvons également mentionner un autre camp de concentration, il s'agit d'Abu-Zaabal où les détenus subissaient un sort plus épouvantable. L'administration de cette prison les tourmentait, sans prendre en considération leurs revendications. L'autorité militaire a de même fondé une autre prison à l'Oasis de Kharga où les détenus politiques menaient une vie inhumaine. C'est ce qui a poussé des intellectuels français parmi lesquels se trouvaient François Mauriac et Jean Paul Sartre, à réagir en envoyant une lettre à Abdel Nasser. Nous en citons cet extrait: "Nous sommes informés que plusieurs centaines de détenus politiques ont été déportés à l'Oasis de Kharga, où ils se trouvent dans des conditions inhumaines. [...] Nous souhaitons que soient prises aussitôt que possible les mesures nécessaires pour que ces déportés soient replacés dans des conditions normales de détention" (Éditions Esprit, p. 621).

Après la mort d'Abdel Nasser, le président Sadate a attaqué sa politique. Pour mettre à l'écart tous ses ennemis, il a fait "arrêter les barons les plus à gauche du régime qui pensaient le réduire à une marionnette" (Solé, 2014, p. 54). Après avoir infligé à Israël sa première défaite en 1973, il est devenu plus

(Prison et liberté d'après ...) Dr. Ayman El Ghandour 
puissant, surnommé dorénavant le héros du franchissement. Son autorité est devenue absolue. Il a adopté l'ouverture économique qui a creusé la lacune entre les riches et les pauvres. Face à l'injustice sociale, ces derniers ont révolté en 1977; il s'agit d'une émeute de la faim "qui fera officiellement 79 morts, 800 blessés, et plus d'un milliard de livres de dégâts" (Desjardins, 1981, p. 442).

En 1977, Sadate est parti pour Israël et a prononcé un discours devant la Knesset. Lui et le premier ministre israélien ont signé les accords de Camp David en 1978. Un an après, l'Égypte a récupéré le Sinaï, Sadate et Begin ont obtenu le prix Nobel de la Paix. Cependant les défenseurs de l'affaire palestinienne n'ont cessé de critiquer le président égyptien et ont considéré cet accord comme une menace permanente au front arabe uni. Sadate a dû encourager les islamistes face à ses ennemis intérieurs, ceux de la gauche. Mais il a commis une erreur en tenant le pape Chenouda pour un comploteur ayant pour but de "fonder un État copte ayant Assiout pour capitale" (Solé, 2013, p. 260). De plus, il l'a enfermé dans un couvent du désert. Ceci a contribué à la montée du courant islamique dont les membres fanatiques ont brûlé des églises, pillé les magasins et les maisons des coptes. La dictature de Sadate s'est traduite en septembre lorsqu'il a lancé "une offensive contre les intellectuels et les activistes de tout le spectre idéologique. [...] Au total, près de 1600 personnes sont arrêtées" (Khallaf, 2015, p. 61), parmi lesquelles se trouvait notre écrivaine Latifa Al-Zayyat. Un mois après, Sadate a trouvé sa fin, assassiné par Khalid Islambouli, membre au mouvement de Jihad islamique.

Quant au contexte politique du Maroc, les années $60 \mathrm{du}$ $\mathrm{XX}^{\mathrm{e}}$ siècle ont annoncé la mort de Mohamed $\mathrm{V}$ et la venue de

(Prison et liberté d'après ...) Dr. Ayman El Ghandour 
Hassan II, "intronisé le 3 mars 1961" (Mésavage, 2004, p. 184). Son règne qui a duré 38 ans, est le plus terrible dans l'histoire marocaine. Un an après sa venue, il a fait rédiger la constitution 62, cause essentielle d'une opposition incessante entre la famille royale et le bloc national, composé de partis politiques. En vertu de cette constitution, le roi a dominé tout: il "nomme le premier ministre et les ministres (art. 24). [Il] préside le conseil des ministres (art. 25). [Il] promulgue la loi (art. 26). [...] Le Roi est le chef suprême des forces armées royales (art. 30)" (mjp.univperp.fr/constitution/mai1962-htm.). Il présidait de même le conseil supérieur dans tous les domaines. Pendant son règne, connu par sa corruption, des émeutes de la faim ont éclaté. Balta dit: "Mars 1965: de sanglantes émeutes éclatent à Casablanca et s'étendent à d'autres villes" (1999, p. 28). C'est une époque de peur, de tension et de mutisme. Pour maintenir son pouvoir, le roi a essayé de faire des concessions et d'établir un climat démocratique mais cette démocratie était illusoire. Sous prétexte de redresser les partis stériles, il a annoncé une nouvelle constitution en 1970 ayant pour conséquence: des élections truquées et un parlement fragile. Ceci a aggravé le mécontentement populaire. Le 10 juillet 1971, le palais de Skhirat était témoin d'un coup d'État, organisé par un lieutenant dissident qui s'appelle Mohamed Ababou. Ce dernier a demandé aux cadets d'Ahermoumou de délivrer le roi, entouré par des traitres dans son palais. Ce n'est pas vrai parce que ceux-ci ont été invités par Hassan II. "Pendant trois heures, Skhirat ne fut que confusion sanglante. Les tirs d'intimidation s'étaient vite mués en tirs à tuer" (Perrault, 1990, p. 129). Le roi a réussi à échapper au massacre; il a condamné 28 officiers et les a envoyés au bagne de Tazmamart. Cachée et située au sud-est du Maroc, cette prison est devenue le symbole de la dictature qui dominait ce pays aux années 70. Aux

(Prison et liberté d'après ...) Dr. Ayman El Ghandour 
dires de Perrault: "Le chiffre exact des emmurés de Tazmamart reste inconnu" (p. 273).

Le 16 août 1972, un autre putsch a eu lieu. Coalisé avec Moulay Abdallah, le général Oufkir a voulu éliminer le roi marocain. "Le Boeing qui ramène Hassan II de Paris est pris en chasse par des F-5 de l'armée. Malgré les tirs de roquettes qui blessent des passagers, le roi refuse de se poser à la base de Kénitra et atterrit à Rabat" (Balta, 1999, p. 29). Le souverain a convoqué le général Oufkir au palais royal. Quelques heures après, on a annoncé le suicide de ce dernier. Sa femme et ses enfants ont été conduits au désert; ils sont restés vingt ans dans des prisons secrètes: Tamattaght, Bir-Jdid.

La situation va de mal en pis avec les années de plomb (1977-1990) pendant lesquelles la monarchie était intransigeante. Celle-ci a essayé sans cesse d'imposer aux opposants sa politique répressive. Les années 90 ont témoigné d'une amélioration démocratique, particulièrement après la parution d'un livre remarquable, intitulé Notre ami le roi; Gilles Perrault y a dénoncé tous les abus de Hassan II. Ce dernier a commencé à écouter l'opposition et à libérer tant de prisonniers politiques.

Après avoir abordé le contexte politique en Égypte et au Maroc, il nous faudrait analyser les trois récits de notre corpus, déterminer leur nature et les motivations qui ont poussé les trois écrivains à les écrire.

\section{Du récit factuel au récit fictionnel}

Commençons avec Latifa Al-Zayyat qui a écrit Hamlat Taftish, ouvrage publié par Dar Al Hilal en 1992, traduit en français par Richard Jacquemond en 1996. Cette traduction qui a

(Prison et liberté d'après ...) Dr. Ayman El Ghandour 
pour titre Perquisition, nous prépare à attendre une action policière; il s'agit de la détention que l'auteure a décrite en détail. La prison est ici réelle et allégorique: la prison de la maison de son enfance, celle de la maison conjugale et enfin celle du gouvernement. Le personnage de Latifa est présenté comme une femme "face à un pouvoir doublement patriarcal: celui d'une société encore largement fondée sur l'homme, et celui d'un régime politique établi sur une figure autocratique" (Hallaq, 1996, p. 30). Elle annonce la mort de son frère au début du roman qui s'achève avec son emprisonnement en 1981. Bien qu'il n'y ait aucun lien entre les deux événements, l'auteure les tisse, l'un à l'autre de manière à dire que l'expérience personnelle glisse dans l'expérience publique et vice versa; elle y narre son existence féminine, son mariage, son divorce, la défaite de 67 et le triomphe d'octobre. Elle s'avère une militante intellectuelle qui "ne veu[t] pas avoir une mort négative"1 (Al-Zayyat, 1996, p. 76).

Quant à La prisonnière, cet ouvrage est un récit de témoignage où Malika Oufkir raconte sa détention et l'expérience vécue pendant son enfance. En l'écrivant, l'auteure déclare sa protestation "contre l'arbitraire de la punition royale infligée à sa famille, la prison pendant vingt ans parce qu'elle s'appelle Malika Oufkir, fille du général Oufkir" (Farhoud, 2008, p. 167). Elle divise son récit en deux parties dont la première a pour titre "L'allée des princesses"; elle y narre son enfance et ses souvenirs au harem royal, exprime ses sentiments lorsque le roi Mohamed V l'a arrachée à sa famille pour devenir compagne de sa fille. Agée de 15 ans, Malika a quitté le palais, revenant à sa maison familiale. Quelque temps après, son père a voulu venger ses amis, exécutés après le putsch de 1971; il a trouvé sa fin après avoir échoué à renverser le roi Hassan II. Ce dernier a donné ses ordres

$$
\text { ' - " لا أريد أن أموت موتأ سلبياً". }
$$

(Prison et liberté d'après ...) Dr. Ayman El Ghandour 
d'enfermer la famille Oufkir. La narratrice relate dans la deuxième partie, leur emprisonnement qui a duré vingt ans aux bagnes de Tamattaght et Bir-Jdid. Le récit s'achève avec leur fuite et leur liberté. Malgré leur détention, ils se trouvent plus mûrs et plus puissants. Rappelons ici les paroles de Malika: "La souffrance peut être un grand enrichissement" (L'établissement détention de Montréal, 1999).

Dans Cette aveuglante absence de lumière, Salim, un détenu, raconte sa vie carcérale au bagne de Tazmamart. A la demande de ses dirigeants, il est allé au palais de Skhirat tout en ignorant la nature de sa mission. Il découvre qu'il a contribué dans un complot contre le roi; il s'agit du coup d'État de 1971. Arrêté, il a passé 18 ans dans la prison, victime d'une mort lente. Le héros dit: "Depuis la nuit du 10 juillet 1971, je n'ai plus d'âge. Je n'ai ni vieilli, ni rajeuni. J'ai perdu mon âge" (2001, p. 14). Il nous présente son parcours de l'école militaire jusqu'au bagne de Tazmamart tout en passant par la prison de Kénitra où il est resté quelques ans. A travers ce roman, l'auteur a pu révéler la souffrance et la douleur infinie que le souverain marocain avait infligées aux détenus politiques dont les uns se sont livrés à la mort. Cependant certains d'entre eux ont pu supporter leur détention jusqu'au bout et ont obtenu leur liberté après l'intervention des organisations des droits de l'homme. Avec cette fin, Ben Jelloun a réussi à mettre à nu la violence d'État et ses pratiques répressives, dénonçant "la dépendance $d u$ système judiciaire de l'État et les aberrations qui en découlent" (Attafi, 2012, p. 195).

Après ce résumé, il nous semble que Perquisition et $L a$ prisonnière sont des récits autobiographiques où les deux auteures remontent aux temps révolus et essayent de reconstituer leur

(Prison et liberté d'après ...) Dr. Ayman El Ghandour 
propre présence. Chacune d'entre elles est à la fois auteure, narratrice et héroïne, conformément aux dires de Philippe Lejeune: "Pour qu'il y ait autobiographie (et plus généralement littérature intime), il faut qu'il y ait identité de l'auteur, du narrateur et du personnage" (1996, p. 15). Bien que Malika confie à Michèle Fitoussi de l'aider à écrire sa biographie, celle-ci affirme dans la préface de La prisonnière qu'elle n'y a introduit aucune imagination; elle dit: "J'ai transcrit ce que j'ai entendu au fil des jours, le témoignage brut de Malika" (1999, p. 17). Fidèle, Fitoussi a parlé au nom de Malika qui n'avait pas encore commencé sa carrière littéraire. De son côté, c'est Latifa AlZayyat qui a écrit son autobiographie, transcrivant immédiatement son expérience aux lecteurs; elle avait tous ses outils. Avec ou sans intermédiaire, ces deux ouvrages appartiennent à l'écriture de soi où se côtoient la réalité personnelle et la réalité historique. Les deux écrivaines y présentent un témoignage oculaire en relatant les événements qu'elles ont vécus. Rappelons ici les dires de Dulong: "Le témoignage oculaire est un récit autobiographique certifié d'un événement passé" (1998, p. 43). Ceci rend leurs récits plus crédibles et influence le lecteur qui leur accorde toute sa confiance. Celui-ci se fie aux dires du témoin historique avec qui il signe un pacte de crédibilité.

En évoquant leur vie et le passé, Latifa et Malika ont utilisé le "je" du récit autobiographique. Cette narration à la première personne rend leur écriture crédible et authentique. La crédibilité remonte à la nature de l'écriture de soi où l'autobiographe "se prend lui-même pour objet d'un texte qu'il écrit" (Gusdorf, 1990, p. 122). Cependant ce dernier a le droit de ne pas se résumer à narrer seulement sa vie; il peut mêler parfois un imaginaire à son récit. Ceci paraît évident avec Al-Zayyat qui a essayé de cacher ses secrets et de mettre à l'écart une partie de sa vie, elle a

(Prison et liberté d'après ...) Dr. Ayman El Ghandour 
présenté des fragments dispersés alors que Malika a poursuivi un ordre chronologique dans son récit.

En fait, c'est le pacte qui sert à différencier l'autobiographie du roman fictionnel, à indiquer les liens entre l'écrivain et ses lecteurs. C'est ce qu'affirme Paul Ricoeur: "La distinction entre récit historique et récit de fiction réside dans la nature du pacte implicite" (2000, p. 339). Ce pacte est autobiographique lorsqu' il montre la relation entre la biographie de l'écrivain et les faits qu'il a mentionnés dans son récit; il est fictionnel lorsqu'il est axé sur le côté imaginaire, présenté par le narrateur. Aussi la narratologie nous aide-t-elle à remarquer cette différence entre les deux genres. Genette affirme que "tous les traits distinctifs entre fictionalité et factualité étaient d'ordre narratologique" (1991, p. 88). Il a présenté cinq schémas triangulaires dont nous choisissons les deux convenables aux récits de notre corpus:

A

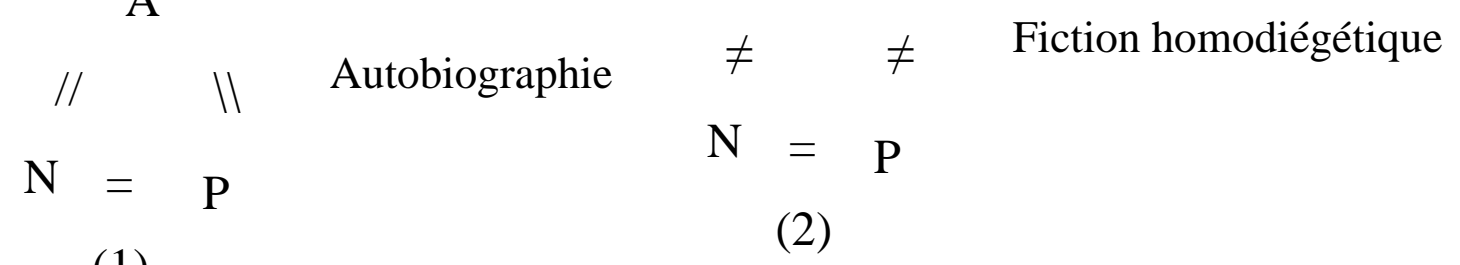

Le triangle (1) montre que l'autobiographie existe à condition que l'auteur, le personnage et le narrateur soient identiques; c'est le cas dans Perquisition et La prisonnière où l'auteure donne son nom à un personnage et lui confie la narration; alors que le triangle (2) annonce que le récit est fictionnel et homodiégétique lorsque l'écrivain est dissocié du personnage qui assume la narration du récit. C'est le cas dans Cette aveuglante absence de lumière où Salim est à la fois narrateur et personnage fictif, n'ayant aucune relation avec l'auteur Tahar Ben Jelloun.

(Prison et liberté d'après ...) Dr. Ayman El Ghandour 
Le lecteur de Cette aveuglante absence de lumière se retrouve dès le début devant un ouvrage qui relate un vrai événement, mais filtré par le style poétique de Ben Jelloun. Celuici annonce la présence d'un tiers en dédiant son récit à Aziz Binebine: "Ce roman est tiré de faits réels inspirés par le témoignage d'un ancien détenu du bagne de Tazmamart. Il est dédié à Aziz ainsi qu'à Réda, son jeune fils, lumière de sa troisième vie" (2001, p. 7). La présence de ce tiers nous rappelle celle de Fitoussi dans l'œuvre de Malika Oufkir. Il est évident que les deux écrivains ont coopéré avec un tiers afin de rédiger leurs récits, mais d'une manière tout à fait différente; d'une part, Malika a collaboré avec Fitoussi, cette journaliste qui n'était jamais emprisonnée, elle est un témoin neutre et indirect. Son rôle se borne à écouter Malika, accueillir son témoignage et l'aider à organiser ses idées pour en faire un roman significatif; d'autre part, la dédicace que Ben Jelloun a adressée à Aziz Binebine, annonce la présence d'un tiers, mais elle ne montre pas la nature de cette collaboration. Contrairement à Fitoussi, Ben Jelloun a recueilli le témoignage d'Aziz non pas pour l'aider à documenter ses souvenirs, mais dans le but d'en faire un ouvrage romanesque. Écoutons Ben Jelloun le dire, lui-même: "Dans mon roman, il y a $20 \%$ de réalité et $80 \%$ d'imagination" (Haubruge, 24/1/2001). Le témoignage d'Aziz constitue ainsi le matériau de base auquel Ben Jelloun a ajouté un écart fictionnel pour construire son roman. A travers ses procédés stylistiques, il a essayé de transmettre aux lecteurs, le despotisme qui a frappé ces détenus politiques, impliqués involontairement dans l'attentat de Skhirat. Il a réussi à se dégager du pacte de vérité qui distingue la littérature testimoniale tout en consacrant une part considérable au pacte romanesque. Celui-ci est marqué par "deux aspects: pratique patente de la non-identité (l'auteur et le personnage ne portent pas le même nom), attestation de fictivité (c'est en général le sous-

(Prison et liberté d'après ...) Dr. Ayman El Ghandour 
titre roman qui remplit aujourd'hui cette fonction sur la couverture)" (Lejeune, 1996, p. 27). Dans son récit, nous trouvons tous les aspects du pacte romanesque: le mot roman est écrit sur la couverture, la dédicace adressée à Aziz, le réel mêlé au fictif, sans oublier que le narrateur s'appelle Salim. Tout cela rend le récit fictif, malgré qu'il présente un témoignage réel. Le lecteur n'est pas sûr si l'histoire narrée est celle d'Aziz Binebine. La vérité et la fiction s'entremêlent si bien qu'il est difficile de séparer l'une de l'autre.

En fait Ben Jelloun a voulu faire un récit fictif et son désir a été renforcé par celui de Binebine. Dans son interview avec Haubruge, L'auteur, lui-même, dit franchement: "Aziz m'a dit qu'il tenait à ce que mon livre soit un roman et pas un document ni un témoignage. Ce qui m'arrangeait bien sûr, car je voulais la même chose" (2001). La fiction n'est pas gratuite. Ben Jelloun l'a utilisée non pas pour trahir la réalité, mais pour influencer les lecteurs et faire vibrer leur sensibilité. L'intérêt est ainsi réciproque entre Ben Jelloun et Binebine; l'un était obligé de s'appuyer sur l'expérience carcérale d'un rescapé pour acquérir une légitimité en abordant la détention au bagne de Tazmamart; l'autre avait besoin d'un écrivain habile à transcrire fidèlement sa souffrance et celle de ses amis.

\section{La vie carcérale}

La prison est un lieu fermé et infernal. Pour les détenus, elle constitue un double isolement; elle les met à l'écart du reste de l'univers, les sépare les uns des autres à l'aide des barrières intérieures. Pour soutirer des informations, les enquêteurs adoptent l'isolement qui influence passivement le moral des prisonniers et les rend inaptes à réfléchir; ils se trouvent dépossédés de leurs gestes et de leurs paroles. S'ajoutent à ces

(Prison et liberté d'après ...) Dr. Ayman El Ghandour 
conditions pénibles, la surveillance permanente, le manque des aliments et des médicaments. Mentionnons ici les dires d'Ekotto: "Les détenus sont en prison, non pas pour être complètement éliminés, comme le voudraient les fanatiques de la défense de la société, ni pour être amendés, comme le souhaitent les rêveurs de la réinsertion, mais pour souffrir" (2001, p. 136). Bref l'emprisonnement a des effets considérables sur le corps du détenu, ses traits moraux et psychiques.

La prison politique n'est que le résultat de la violence de l'État, pratiquée en cachette. Tout État croit à la légitimité de la violence qui l'aide à maintenir son ordre interne. Cependant, il ne tarde pas à utiliser la violence illégitime face aux émeutes et aux manifestations afin d'éviter des confrontations coûteuses.

Latifa Al-Zayyat, cette militante de gauche, était victime de la violence de l'État, emprisonnée deux fois en 1949 et en 1981. Pour elle, "la lutte politique était toujours un combat contre le gouvernement égyptien, sa police et son armée" (Fahndrich, 1999, p. 81). Elle ne se contente pas d'aborder implicitement cette violence à travers son roman La porte ouverte, mais elle a documenté son expérience carcérale dans Perquisition; elle nous y a fait voir des policiers faire feu sur des manifestants à Mansoura, ainsi que des cadavres flotter sur le Nil. En voyant cette scène sanglante, la narratrice dit: "Je tue l'enfant en moi et la fillette mûrit trop vite, endurcie par un savoir qui dépasse les limites de la maison" ${ }^{2}$ (1996, p. 42).

Latifa est arrêtée par le régime de Sadate qui a essayé de mettre fin à tous ses opposants: journalistes, étudiants, intellectuels et professeurs universitaires. Ce qui nous frappe ici,

$$
\text { × - " أسقط الطفلة عني و الصبية تبلغ قبل آوان البلوغ مثخنة بمعرفة تتعدى حدود البيث". }
$$

(Prison et liberté d'après ...) Dr. Ayman El Ghandour 
c'est la venue de "dix soldats armés jusqu'aux dents pour surveiller une femme de cinquante-huit ans" ${ }^{3}$ (Al-Zayyat, 1996, p. 82). Ils l'ont conduite à la prison de Kanater, accompagnée d'autres détenues politiques telles Amina Rachid et Nawal El Saadawi qui a écrit Douze femmes dans Kanater (1984). L'auteure affirme ce que nous avons déjà dit: il s'agit du despotisme de Sadate et son "ordre d'arrestation de mille cinq cents opposants au traité de Camp David"4 (p. 82)

Frappée de l'abîme entre le réel qu'elle vit et le rêve qu'elle souhaite, la narratrice a mis l'accent sur la prison, lieu de misère et de violence. Les détenus y mènent une souffrance physique et psychique; l'incarcération leur représente un stress et un déséquilibre où la force est à la base de leurs relations; ils sont accablés par un état d'inquiétude, d'insécurité et d'impuissance ayant des effets passifs. Lors de son premier emprisonnement, la jeune héroïne a "découvert une des dimensions des menaces en entendant les gémissements des tortures au gouvernorat d'Alexandrie" $^{5}$ (1996, p. 93). Pendant sa deuxième détention, la narratrice a présenté la prison comme un cauchemar où elle était égarée, privée de tout; elle a mis l'accent sur les murs si hauts et infranchissables qu' "au-delà, on ne devinait rien, ni de près ni de loin, comme s'il n'y avait que le néant après ce mur" ${ }^{\prime 6}$ (p. 87). Ces murs découragent les détenus pour qu'ils se soumettent à l'administration et ne pensent jamais à la fuite. Persuadée que la prison symbolise la violence inhumaine, la narratrice la considère dans un autre endroit, comme un "des moyens de priver l'homme de son humanité et de son pouvoir de réflexion critique" ${ }^{7}$ (p. 95).

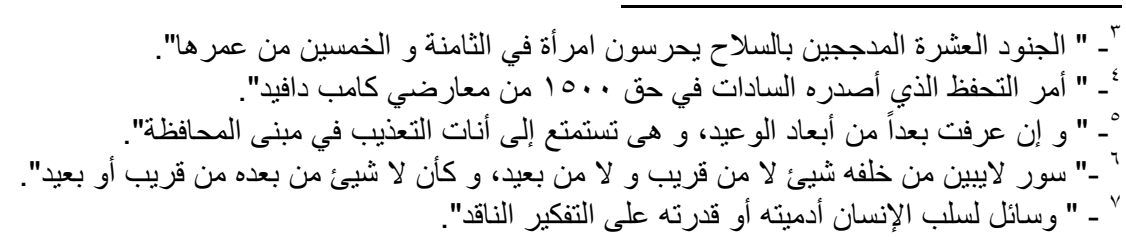

(Prison et liberté d'après ...) Dr. Ayman El Ghandour 
Latifa ne tarde pas à dénoncer l'administration pénitentiaire qui méprise totalement les détenus politiques, néglige leur nourriture et leurs médicaments, les intimide et les tourmente cruellement. Elle annonce que "l'oppression qu'exerce l'autorité et

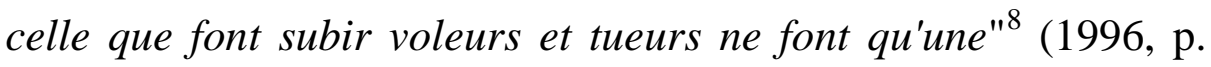
116). Elle compare ainsi l'autorité au chef d'une bande de voleurs. Cette croyance la rend plus forte que jamais. Nous l'avons vu aider ses amies, cacher le poste de radio et les notes écrites d'Amina Rachid, lutter contre l'administration afin de "parvenir au minimum de décence de toute vie humaine" ${ }^{9}$ (p. 113). Elle nous annonce catégoriquement sa métamorphose: "La prison transforme les gants de soie blancs et doux en gants de boxe" ${ }^{10}$ (p. 112).

La narratrice a évoqué une des mesures répressives de l'administration carcérale; il s'agit de la fouille, même dans une latrine où les gardiennes ouvrent les portes et insultent les prisonnières. Nous la trouvons "terrifiée, devant une femme aux yeux difformes, à la poitrine et aux fesses plates" ${ }^{11}$ (1996, p. 115). Celle-ci la fait se rappeler Raya et Sekina, ces deux criminelles qui ont tué tant de victimes et dont elle avait peur pendant son enfance. Mais "l'enfant qui trouvait refuge des maux du monde dans les bras de sa mère"12 (p. 115), est maintenant fragile, accompagnée d'autres prisonnières, incapables de faire face aux gardiennes brutales.

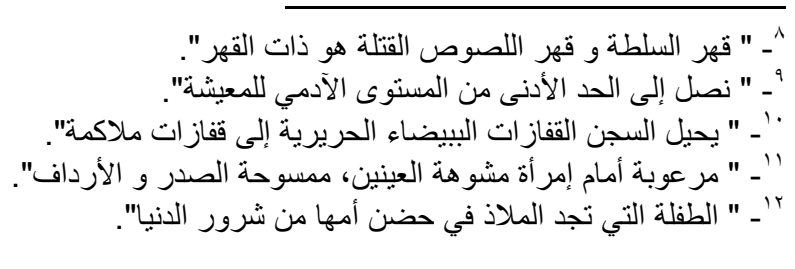

(Prison et liberté d'après ...) Dr. Ayman El Ghandour 
Dans Perquisition, Latifa a présenté d'autres formes de la prison: celle de la famille où la petite fille était inférieure à son frère; elle " $n$ ' [a] jamais connu le luxe d'inviter [ses] camarades de classe à la maison"13 (p. 30); celle du mariage où le mari autoritaire lui a dit: "C'est moi qui t'ai faite"14 (p. 45); elle était marginalisée, n'ayant aucune existence, chargée seulement de ses tâches ménagères. C'est ce qu'elle a déjà abordé dans La porte ouverte, mettant l'accent sur la résistance de Layla aux traditions sociales, aux mariages forcés qui conduisent au malheur. Cette prison est autant difficile que celle de l'État. La narratrice avait du mal à obtenir sa délivrance et son divorce. Tout dépend de la volonté de son mari. Mentionnons ici ses paroles significatives: "un mois à préparer l'entrevue du divorce, par la prière et la douceur par l'entremise des parents, des proches et des amis, par la menace $^{115}$ (p. 47).

Quant à Malika, la détention de sa famille constitue le noyau de son autobiographie; elle y présente son témoignage en vue d'oublier un passé tragique et de restaurer une image idéale de son père et de sa famille incarcérée. Sortant de sa réclusion, elle écrit son expérience pour "retrouver une place dans le corps social et une parole" (Ricœur, 2000, p. 454). Elle considère ainsi l'écriture comme un espace de liberté et un moyen de s'évader du désespoir.

La narratrice affirme dès le début que son incarcération a commencé dès sa prime enfance. Adoptée par Mohamed V à l'âge de cinq ans, elle nous transmet sa tristesse et son amertume: "M'arracher à ma mère, c'était m'arracher à la vie. J'ai pleuré,

$$
\begin{aligned}
& \text { "َ"- " لم أتمتع برفاهة استضافة زميلاتي في البيث". }
\end{aligned}
$$

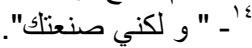

$$
\begin{aligned}
& \text { "' " " لي شهر أدبر الطلاق، بالرجاء بالحسنى، بتوسط الأهل و الأقارب و الأصدقاء بالتهديد". }
\end{aligned}
$$

(Prison et liberté d'après ...) Dr. Ayman El Ghandour 
hurlé, trépigné" (1999, p. 34). Elle a passé dix ans au palais royal, "presque cloîtrée" (p. 36). Elle était frappée en voyant quatrevingts concubines vivre auprès du roi dans le but de lui faire plaisir. Comme sa mère lui manquait, elle souffrait sans cesse au point qu'elle a voulu se suicider. Adolescente, elle avait besoin d'elle afin de comprendre ses transformations physiques. Malika et ses compagnes étaient obligées d'écouter le grand mollah, chargé de leur expliquer l'éducation sexuelle. Il leur "apprenait que les femmes ne sont que séduction et soumission, que leur corps sert avant tout à satisfaire les désirs de l'homme" (p. 82). Leur valeur réside ainsi dans leur féminité. Tel décor, la narratrice oppressée dit: "Plus je grandissais et plus j'avais le sentiment d'être prisonnière" (p. 86, 87).

Le roi Hassan II ne s'est contenté de faire exécuter le général Oufkir, mais il a décidé d'anéantir sa famille et transformer ses membres en fantômes, déformant leur présent et leur futur. A Tamattaght, la famille composée de neuf personnes, était enfermée dans un palais emmuraillé; ils ont découvert que ce bagne maudit "a été construit sur un cimetière" (Oufkir, 1999, p. 170). Au milieu du silence et de l'obscurité, ils avaient le sentiment d'être enterrés vivants. Cependant on les a conduits au bagne de Bir-Jdid et les a emprisonnés dans des cellules humides, insalubres, habitées de scorpions, serpents, mouches, punaises et moustiques. Dans cette ambiance abominable, la narratrice crie: "Enfermés nuit et jour, séparés, maltraités, rien désormais ne nous rattachait plus à notre ancienne vie" (p. 202). A toutes ces conditions pénibles, s'ajoutent la barbarie carcérale et la nourriture si insuffisante que l'héroïne dit: "La faim humilie, la faim avilit. La faim vous fait oublier votre famille, vos amis, vos valeurs. La faim vous transforme en monstres" (p. 212).

(Prison et liberté d'après ...) Dr. Ayman El Ghandour 
Autre souffrance pèse sur tous les membres de la famille Oufkir, c'est la surveillance ayant pour but de les priver de leur intimité et de les décourager. Ils se trouvent toujours face à celui qui les observe sans être vu. Paralysée de cette situation pénible, Malika dit: "Je souffrais pour nous tous, dépossédés de liberté et d'espoir" (p. 185). Leur sentiment d'être surveillés tout au long du temps, fait émerger chez eux une terreur physique et psychologique. Tout ce qu'ils font, est épié. Même lors de leur fuite, tout le Maroc leur paraît un espace carcéral. Le cuisiner que Malika a rencontré, affirme que "Ce pays devient un véritable État policier" (p. 314). Au bagne, la situation de la famille Oufkir va de mal en pis, particulièrement Malika, incapable de vivre une belle histoire d'amour. Plus les jours passent plus elle périt tout en perdant sa beauté. Celle-ci a fait Cappaccio, un des gardes, se coller contre elle. "Son corps [l'] oppressait" (p. 241). Bien qu'elle réussisse à subir son assaut, elle reste paralysée, ayant peur du viol, des coups et de l'humiliation.

De sa part, Tahar Ben Jelloun donne la parole à Salim qui narre à la première personne sa détention et celles de ses compagnons. Grâce à cette narration homodiégétique, Salim nous place à l'intérieur du récit et relate tout ce qui se passe autour de lui. Ces détenus dont les histoires s'entrecroisent, sont envoyés au bagne de Kénitra, transférés plus tard à Tazmamart, lieu inconnu. "Transfèrement non seulement géographique mais chronologique: c'était comme s'ils avaient été retirés du siècle par la main royale" (Perrault, 1990, p. 267). Bien qu'ils y passent dix ans, ils ne sont pas libérés. Face à des geôliers cruels, ils comprennent que ce bagne nocturne est leur tombeau. L'auteur donne la parole aux détenus pour qu'ils témoignent de leur vie carcérale et inhumaine. Le désespoir les pousse à se suicider. Mentionnons à titre d'exemple Hamid qui "se cogna plusieurs fois la tête contre

(Prison et liberté d'après ...) Dr. Ayman El Ghandour 
le mur" (2001, p. 19). Certains d'entre eux sont morts à la suite d'autres causes: maladie, folie, piqûres de scorpions. Malgré leur tragédie, les détenus de Tazmamart nous choquent lors de l'enterrement de l'un d'entre eux. Nous les voyons contents grâce à leur contact avec l'air et la lumière.

Le bagne de Tazmamart est tout à fait différent d'autres prisons. Il représente pour les détenus marocains ce que représente Auschwitz pour les Juifs. Ils s'y trouvent face à l'humiliation et à l'obscurité écrasante qui domine sans cesse toutes les cellules. L'administration carcérale leur annonce qu'ils sont "condamnés aux ténèbres éternelles" (Ben Jelloun, 2001, p. 75). La nuit les entoure et les pénètre à tel point que Salim dit: "La nuit nous habillait" (p. 10). La privation de lumière est ainsi leur première sanction. Ben Jelloun insiste à présenter ce bagne comme un outil de violence, adressé contre les détenus politiques, non pas comme un lieu visant à redresser leur comportement. Ceci paraît évident à travers le personnage de Salim, victime de la mort lente, dont le "corps était cousu de cicatrices" (p. 217). L'administration carcérale leur impose l'isolement et la solitude afin de les obliger à une soumission totale. Ces conditions pénibles sont aggravées de plus en plus par la présence des gardes intransigeants, particulièrement le directeur du bagne qui reste invisible jusqu'au bout. Il est envoyé pour rendre la détention plus terrible et plus infernale. Nous le voyons "donner l'ordre à un quidam d'aller ramasser des scorpions et de les introduire dans [leur] fosse" (p. 56). Ces scorpions symbolisent non seulement son agressivité vis-à-vis des détenus, mais aussi une autorité dictatoriale ayant pour but d'appliquer la violence pour écraser les opposants et assurer la survie du roi.

(Prison et liberté d'après ...) Dr. Ayman El Ghandour 
Le bagne de Tazmamart est si enfermé qu'il empêche les détenus de distinguer le jour de la nuit, de déterminer exactement le temps. L'obscurité dominante active leur douleur et les rend plus sensibles à la souffrance. Ceux-ci ont du mal à se déplacer et à tenir débout. Aussi le trou consacré aux toilettes engendre-t-elle une puanteur et des maladies inguérissables. Malgré leur souffrance, un garde les menace en réclamant: "Vous pourriez être utiles: marcher en avant pour baliser la route semée de mines" (Ben Jelloun, 2001, p. 44). En dévoilant tous ces crimes, ce roman s'avère une trace de la violence exercée par une autorité despotique même si celle-ci a fait détruire ce bagne. Le narrateur affirme à la fin du récit: "Mais si des soldats réussissent à effacer les traces du bagne, jamais ils n'arriveront à effacer de notre mémoire ce que nous avons enduré" (p. 215).

Les trois auteurs de notre corpus ont de même évoqué les gardes humains. Ces derniers étaient persuadés que ces détenus politiques n'avaient pas besoin d'un redressement. Dans Perquisition, le personnage de Latifa a pris des aliments d'un soldat "dont la bonté avait fait échouer le plan policier" (1996, p. 92). Aussi Malika nous a-t-elle fait entendre les paroles du capitaine Chafiq: "Massacrer des enfants, c'est au-dessus de mes forces" (1999, p. 254, 255). Il y a d'autres soldats qui n'ont cessé de leur procurer de la viande et des œufs frais. De plus, ils les prévenaient les jours de perquisition. De son côté, Ben Jelloun a indiqué le rôle efficace de M'Fadel qui était intermédiaire entre les détenus et leurs proches; il "porta le bout de papier à la femme de Wakrine" (2001, p. 70). Grâce à ce papier, une militante pour les droits de l'homme a révélé l'état pénible de ces détenus. Ceci a poussé la presse mondiale à dénoncer la politique despotique du roi Hassan II.

(Prison et liberté d'après ...) Dr. Ayman El Ghandour 


\section{A la recherche de la liberté}

Latifa Al-Zayyat ne s'est livrée ni à la violence sympathique ni aux traditions sociales qui ont mis les femmes à l'écart de l'espace public et les ont obligées à rester dans leurs maisons. Amatrice de la liberté, elle avait l'habitude de "dire non à toutes les injustices du monde" (Ayad, 1996). Ici réside la réelle motivation qui l'a poussée à écrire son autobiographie. Elle a voulu outrepasser cette violence, refusant d'être confinée au silence. Ecrire son expérience carcérale et ses problèmes sociopsychologiques lui parait un pas efficace vers l'émancipation des Égyptiennes et l'existence d'une harmonie entre l'égo féminin et l'espace social. Grâce à ses écrits et ceux d'autres compagnes telles Nawal El Saadawi, Amina Rachid et Radwa Ashour, elles ont réussi à pénétrer l'espace public réservé aux hommes et à être la voix des femmes égyptiennes. Celles-ci se trouvent dorénavant comme compagnes et non pas comme subalternes.

Après son entrée à l'université, Latifa a étudié la littérature anglaise qui lui a permis de lire le marxisme; elle a contribué à fonder la ligue des jeunes femmes de l'université et des instituts. Agée de vingt ans, elle est devenue "le porte-drapeau du Comité national des étudiants et des ouvriers" (Hallaq, 1996, p. 30). Elle s'est lancée dans la lutte patriotique pour réveiller le peuple, chercher sa liberté et celle de son pays. Militante, elle a approuvé que la société conservatrice ne pouvait pas entraver les nouvelles voix de lancer des perspectives émancipatrices et de faire face à la domination anglaise. Son engagement lui a coûté cher. Monciaud a montré que Latifa avait subi des insultes "de la part des militants estudiantins intégristes. Ces pratiques diffamatoires sont alors fréquentes contre les femmes investies en politique" (2008, p. 4). Ces insultes ne l'ont pas empêchée d'aller jusqu'au bout, devenant une des fondatrices du comité de la défense de la culture

(Prison et liberté d'après ...) Dr. Ayman El Ghandour 
nationale (1979-1996). Contente de sa lutte pour la liberté et de son insertion sociale, la narratrice exprime sa colère: "Ce que je n'ai jamais cessé de désirer s'était réalisé: j'étais devenue une partie du tout" ${ }^{16}$ (1996, p. 35).

Face aux traditions conservatrices qui marginalisent les jeunes filles, la petite Latifa aime monter sur la terrasse où elle n'est pas surveillée. Elle exprime sa joie: "Sur la terrasse, je saute à la corde, et mes bonds s'élèvent toujours plus jusqu'à toucher le ciel de ma tête, sans être vue ou restreinte par personne ${ }^{17}$ (1996, p. 22). Elle danse, chante et lance sa voix que le vent emporte au plus loin. Là, la fille dépasse les contours de sa maison familiale et observe attentivement la ville de Damiette. Nous la voyons dans autre endroit, courir dans le jardin de sa maison, sauter de nouveau en l'air. Bras et pieds nus, elle danse follement sans répondre aux ordres de sa mère qui "se perdent, couverts par les cris de joie folle de la fillette" ${ }^{18}$ (p. 26). Même après son mariage, elle refuse de se soumettre à un mari autoritaire. Divorcée, elle dit avec fierté: "J'étais désormais en mesure de dire "Non, ça suffit", et de ne pas enterrer ces mots dans un sommeil de mort" ${ }^{19}$ (p. 47). De plus, elle lie sa liberté à celle de son pays; elle semble effondrée après le désastre d 67 et la prise du Sinaï par les Israéliens; elle exprime sa déception et son découragement en confirmant: "La défaite de 1967 m'avait dévastée" ${ }^{20}$ (p. 51).

Pour Malika Oufkir, elle a conçu que son identité ne se réaliserait que dans les rues. Pour que cette identité devienne

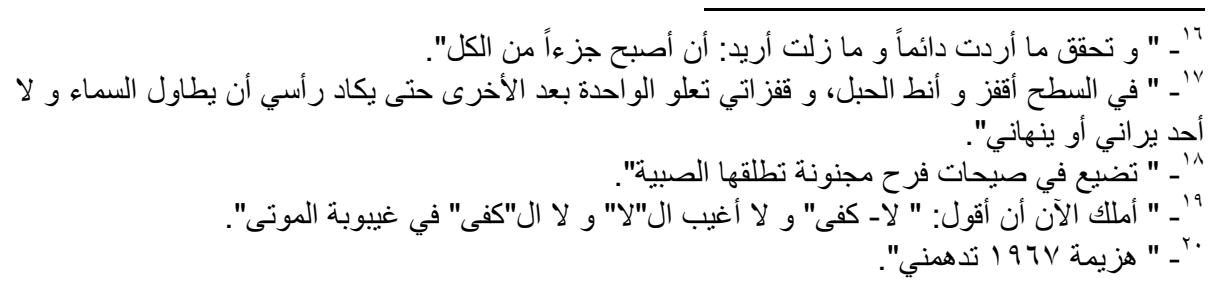

(Prison et liberté d'après ...) Dr. Ayman El Ghandour 
tangible, la fille du général Oufkir a décidé d'échapper au bagne et de défendre le nom de son père de tout ce que Hassan II lui a infligé. Grâce à l'évasion de Malika, accompagnée de ses deux frères et à leur contact avec des journalistes français, le président Mitterrand leur a envoyé un message d'encouragement: "Vous devez être très fiers de vous [...] Vous resterez les seuls qui n'ont pas baissé le bras." (Oufkir, 1999, p. 329). Ceci a permis au reste de la famille de se libérer. Cependant ils ont passé cinq ans à Marrakech, assignés à résidence. Ils ont obtenu leur pleine liberté en 1991, après la parution de Notre ami le roi de Gilles Perrault. Après l'évasion de sa sœur Maria vers la France en 1996, Malika a quitté son pays natal, s'installant à Paris où elle s'est mariée avec Eric, un architecte français.

L'amour de Malika pour la liberté et la dignité est fortement tangible dans La prisonnière. Agée de cinq ans, sa mère l'a emmenée au palais royal où l'enfant a rencontré pour la première fois la princesse Lalla Mina, une fillette de son âge. Celle-ci a mordu Malika, choquée par la réaction de sa mère qui lui a ordonné de se calmer. A son tour, elle lui a arraché "la joue d'un coup de dents. La princesse se mit à son tour à hurler si fort que la cour entière se leva" (Oufkir, 1999, p. 33). Contrairement à toute l'audience horrifiée par les paroles de Malika, le roi Mohamed V a annoncé son désir de l'adopter. Bien qu'elle reçoive un enseignement en français, elle était rebelle et insoumise à ses professeurs. La narratrice montre qu'elle "étai[t] rebelle dans l'âme, rétive à toute forme d'autorité" (p. 103); elle ne voulait que quitter ce palais. "Le soir, dans mon lit, dit-elle, je rêvais de liberté" (p. 85). Même après son retour à la maison de son père, elle menait une vie étouffante, surveillée par une escorte. Cependant elle avait l'habitude de sortir en cachette et de danser jusqu'à l'aube, dans une boîte de nuit.

(Prison et liberté d'après ...) Dr. Ayman El Ghandour 
Dans La prisonnière, Malika dénonce la domination virile, refusant de voir la femme soumise à l'homme, même si ce dernier est le roi, lui-même. Au palais royal, elle passe le "temps à réunir les concubines pour tenter de leur ouvrir les yeux sur leur triste sort" (1999, p. 92). Un peu plus loin, elle fait allusion au féminisme et dit avec enthousiasme: "Libre, j'aurais suivi ces femmes-là, j'aurais sans doute milité avec elles" (p. 235). Pour elle, celles-ci ont droit au respect et à l'autonomie; elles sont activistes, habiles à diriger leur vie et à améliorer leur sort. Après sa sortie de la prison, Malika ne change pas d'avis; elle annonce: "Je crois à la liberté féminine, à l'égalité des droits, mais toujours dans le respect, la tolérance et la douceur, jamais dans la violence ou l'agressivité" (2000, p. 46). Son désir d'adhérer à ce mouvement féministe, vient de la souffrance qu'elle a subie soit au palais ou en prison. Victime d'un régime répressif, elle refuse toute agressivité faite aux femmes, basée sur leur infériorité et leur féminité.

En fait, c'est la liberté qui a poussé les Oufkir à fuir la prison. Chacun d'entre eux a enlevé les dalles de sol pour percer un trou de sa cellule à une autre. Malgré leur faiblesse physique, ils ont réussi à creuser un tunnel. L'évasion leur paraît une renaissance. C'est ce qu'affirme la narratrice: "Ces quatre jours de liberté avaient déposé dans nos regards la petite flamme de la vie, que nous croyions pour toujours éteinte" (p. 341). Libérés, ils ne veulent qu'indemniser leur emprisonnement.

Quant à Tahar Ben Jelloun, il est parti pour la France à la recherche de la liberté, perdue au Maroc. Face au régime despotique et aux problèmes socio-politiques de son pays natal, il a semé la révolte et la liberté dans son œuvre. Dans ses Pensées pour la liberté, il nous dit: "La liberté n'est rien si elle ne respire

(Prison et liberté d'après ...) Dr. Ayman El Ghandour 
pas dans le corps et l'esprit de l'homme, de tous les hommes, sans distinction ethnique, religieuse ou géographique" (Citationcelebre le parisien.fr). Il exige que la liberté soit concrète, ressentie de tous les hommes: musulmans et chrétiens, africains, et européens. Dans un entretien avec Valérie Trierweiler, Ben Jelloun a annoncé son soutien pour le printemps arabe et pour les peuples révoltés qui ne voulaient que récupérer leurs droits. Persuadé qu'il a joué son rôle, il dit: "Les écrivains n'ont pas cessé, depuis des années de décrire la pourriture dans ce pays. Leurs écrits ont fait leur chemin et préparé le terrain" (TrierWeiler, 03/07/2011).

Dans Cette aveuglante absence de lumière, Ben Jelloun a essayé de dire l'indicible d'une manière symbolique. Il a mis l'accent sur l'espace carcéral, obscur et abominable où les détenus ne pensaient qu'à la lumière, symbole de la liberté dont ils étaient privés. Face à ce lieu misérable, Salim a voulu faire un trou dans le mur pour que la lumière pénètre dans sa cellule; tout ce dont il a rêvé, c'est l'étincelle que ses "entrailles garderaient protégée comme un secret" (2001, p. 9). En fait, c'est sa mère qui lui a enseigné la dignité et l'amour de la liberté. Ceci parait évident à travers les paroles que le héros lui a adressé: "Jamais tu ne cédais ni ne faiblissais. Ta force de caractère, c'était ta liberté." (p. 72). Elle a refusé de se soumettre à un mauvais mari capricieux. L'enfant qui a découvert en elle un esprit de résistance, a appris comment affronter son adversaire. Il a surmonté les difficultés qu'il avait subies, arrivant au point attendu; il s'agit de sa sortie de la prison. Il dit: "Nous étions le 29 octobre 1991. Je venais de naître" (p. 225). Cette date est significative parce qu'elle symbolise la libération des détenus de Tazmamart. Comme il avait raison, Salim se trouvait tranquille devant le caïd. Au contraire, ce dernier "bafouillait, se trompait, revenait en arrière

(Prison et liberté d'après ...) Dr. Ayman El Ghandour 
à la recherche de ses mots" (p. 224). Cette rencontre a révélé la victoire de la liberté et la défaite du régime despotique sous le règne du roi Hassan II. Ayant peur, l'autorité marocaine a préparé les détenus à bien confronter l'opinion internationale et leur a demandé de ne pas raconter leur expérience carcérale. Elle ne s'est pas contentée de menacer leurs familles, mais aussi elle a totalement détruit la prison de Tazmamart pour effacer ses traces.

Les récits de notre corpus ont révélé que les trois personnages narrateurs étaient attachés à leur liberté. Ils ont réussi à résister à la violence physique et psychique en domptant parfaitement leurs corps et leurs émotions. Celles-ci obligent "l'organisme à fournir un supplément d'énergie pour triompher de l'adversité, pour mieux se défendre" (Regard, 2007, p. 19). Sans elles, l'homme perd sa capacité à affronter les autres. Elles influent directement sur le corps; leurs effets paraissent à travers des changements expressifs comme les larmes et les réactions faciales. Comme l'homme n'est pas de pur esprit, il ressent tout ce qui passe par son corps. Ce dernier "est la marque de l'individu, sa frontière, la butée en quelque sorte qui le distingue des autres" (Le Breton, 2018, p. 8).

Malgré sa lassitude et son épuisement, le personnage de Latifa ne se livre pas au découragement. L'héroïne paraît si forte qu'elle maîtrise ses émotions. Pour elle, "pleurer face aux problèmes était une manière de défaite et de capitulation qu'on ne doit pas afficher devant les autres" ${ }^{21}$ (1996, p. 31). Elle veut cacher le reflet de son état interne, particulièrement ses larmes qui expriment sa faiblesse. Dans un autre endroit, elle n'hésite pas à

$$
\text { بالتالي اعلانه أمام الأخرين" البكاء في وجه المشاكل، و مازلت، نوعاً من الانهز ام و الاستسلام لهذه المشاكل و لا يجوز }
$$

(Prison et liberté d'après ...) Dr. Ayman El Ghandour 
mentionner les dires de Brecht: "Ne manifeste pas d'émotion" ${ }^{22}$ (p. 18). Elle prend de la distance afin qu'elle puisse retrouver son équilibre émotionnel et s'adapter à son entourage. Latifa affirme que "personne ne la priverait de sa faculté de réfléchir, de distinguer le vrai du faux"23 (p. 94).

Dans Perquisition, l'héroïne dénonce l'atmosphère politique, refuse de s'enfoncer dans la déconvenue, essaye de créer de nouvelles motivations. Lors de la prise de son mari, nous la voyons chanter au tribunal: "Demain le printemps refleurira et l'amour reviendra" ${ }^{24}$ (1996, p. 89). Même lors de la défaite de 67, elle répète: "Rien ne me détruira" 25 (p. 67) A peine arrivée à la prison de kanater, elle semble tout à fait séparée de tout ce qui l'entoure, ayant peur d'un destin inconnu. Cependant elle se met à s'acclimater avec la prison tout en maîtrisant son corps au point qu'elle "écoute le sang qui bat dans [ses] veines, bruyante expression de l'amour de la vie et de la volonté de construire une vie meilleure" ${ }^{26}$ (p. 85). Sa réussite à bien contrôler ses émotions la fait observer les moindres détails de la nature, symbole de la vie et de la liberté. La narratrice reste attachée à l'arabe dont les racines se plongent dans la prison alors que ses branches poussent vers le haut; elle jure qu'elle a "entendu la sève courir des racines aux branches et aux fleurs rouges" ${ }^{27}$ (p. 85). Convaincue que ses émotions ne sont pas illusoires, elle réaffirme dans un autre endroit que "le ventre de la terre murmure ses secrets à qui sait tendre le cœur et l'oreille" ${ }^{28}$ (p. 91). Son interaction avec la nature

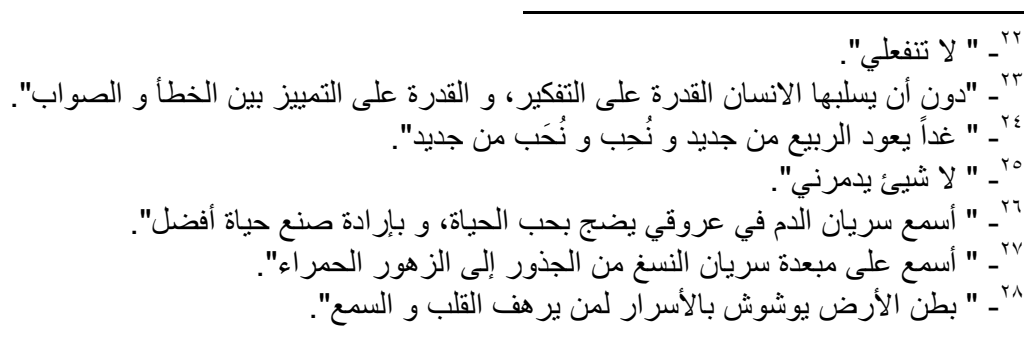

(Prison et liberté d'après ...) Dr. Ayman El Ghandour 
l'a aidée à endurer la souffrance de la prison. Elle nous informe que l'amour humain a transformé ce lieu pénible, "empli de souvenirs amers, en un sanctuaire où [elle a] voulu [se] rendre en pèlerinage $^{\prime 29}$ (p. 62). Ainsi son équilibre intérieur lui a-t-il permis de franchir de l'obscurité à la lumière, de la prison à la liberté.

Quant à La Prisonnière, écrite en témoignage autobiographique, Malika y essaye de retracer son passé et d'évoquer son adolescence et sa jeunesse ratées dans la prison. Reprenons ici ce qu'a dit Farhoud: "Pour Malika Oufkir, écrire et décrire le corps, c'est se libérer de la prison abstraite qui la hante et emprisonne son corps derrière des murs tissés par son amer souvenir pénitentiaire" (2008, p. 56). Son écriture se présente comme une catharsis; elle l'aide à oublier sa longue incarcération. Malika n'est-elle pas victime d'un changement choquant? Après une vie prospère au palais royal, elle se trouve emprisonnée dans un lieu inhumain; il s'agit de la prison qui "permet de voir le réel différemment" (Saraya, 2020, p. 122). Face à cette métamorphose, elle n'a qu'un seul souci: maîtriser ses émotions et vivre dans l'imagination.

Adoptée par le roi Mohamed V et emprisonnée au palais royal, Malika ressemble à une écorchée. C'est ce qu'elle affirme: "En apparence, souriante, joviale, drôle et facétieuse. Mais il suffisait d'un mot, d'un parfum qui me rappelait ma mère, et je me renfermais" (1999, p. 84). Elle ressent une tristesse qui s'approfondit lors de sa détention. C'est une tristesse accompagnée d'anxiété, de colère et de honte de nostalgie. Cependant la jeune fille laisse échapper un sourire artificiel qui ne reflète pas son intériorité afin de ne pas se livrer à des émotions passives; elle

$$
\text { 9" - " أحال بناء مقيناً مليئًا بالذكريات المريرة، إلى كعبة أحج إليها". }
$$

(Prison et liberté d'après ...) Dr. Ayman El Ghandour 
semble plus solide, apte à supprimer tout ce qui la dérange et à éliminer ses désirs. Elle dit: "Notre jeune âge vibrait de passions, de pulsions, de révoltes. Mais il fallait les dompter, apprendre à vivre sans, pour ne plus avoir mal" (p. 238). Elle essaye de se débarrasser de tout ce qui évoque le passé. Mais sa résistance à la nostalgie meurtrière n'est pas facile. C'est ce qu'elle affirme: "J'ai trop de mal a m'arracher à tout ce que j'aimais. J'ai le sentiment d'être emmurée vivante" (p. 153). C'est sa vie passée qui la tourmente et la pousse vers un présent douloureux et un futur ambigu.

Un des moyens efficaces que Malika et sa famille ont adopté pour supporter la souffrance pendant leur emprisonnement, c'est l'humour. Il les a aidés à changer leur perspective et à bien réfléchir. L'héroïne, elle-même, nous affirme que l'humour les " $a$ permis de survivre même, et surtout, dans les moments extrêmes" (1999, p. 229). Dans la prison, ils menaient une comédie noire où ils devaient rire en vue de ne pas tomber en démence. Leur humour est ainsi un outil cathartique; il les aide à oublier provisoirement leur situation pénible. L'humour est ici une sorte de l'intelligence émotionnelle qui leur permet de dompter leurs émotions. C'est pourquoi, l'héroïne qui croit à la puissance de la raison, essaye sans cesse de bien éduquer ses frères et ses sœurs pendant leur détention; elle annonce que l'esprit "nous permettait de tout supporter" (p. 211). C'est le même avis de Latifa AlZayyat que nous avons déjà évoqué.

Nous avons remarqué à quel point Malika et sa famille avaient une stabilité émotionnelle qui leur a permis de résister à la faim, à la douleur et de rester équilibres jusqu'au bout. Ils ont pu dompter leurs corps lors de l'évasion. C'est Raouf qui a choisi l'endroit où ils ont commencé à creuser un tunnel; il "connaissait

(Prison et liberté d'après ...) Dr. Ayman El Ghandour 
au millimètre près la qualité $d u$ sol, sa résonnance, sa sècheresse" (1999, p. 256). Chargés d'une énergie surnaturelle, ils ont achevé leurs tâches sans sentir aucune fatigue. Bien que les pieds de Malika soient sanglants, elle dit: "Je ne sentais même plus la douleur" (p. 339). De plus, elle a pu contrôler son corps gonflé d'œdème afin de s'évader à travers le tunnel. Elle a réussi à mettre toute son "énergie dans l'effort. Ce fut comme un accouchement, une seconde naissance" (p. 272).

De son côté, Tahar Ben Jelloun a essayé d'indiquer à quel point la politique investit le corps humain. Il a pris les détenus de Tazmamart comme échantillon, appliquant ce qu'a annoncé Foucault: "Le corps est aussi directement plongé dans un champ politique; les rapports de pouvoir opèrent sur lui une prise immédiate; ils l'investissent, le marquent, le dressent, le supplicient" (1975, p. 30). L'auteur a mis l'accent sur Salim, un des détenus, à qui il a confié l'acte de narration. Ce dernier est non seulement le personnage focalisateur du roman, mais aussi il s'avère un héros problématique. A travers sa douleur et son expérience carcérale, nous avons constaté de près le despotisme du souverain marocain. Grâce à sa volonté, il a pu résister au châtiment terrible qu'il avait subi.

Dans le roman de Ben Jelloun, les détenus de Tazmamart tiennent la concentration pour une stratégie, visant à atténuer leur souffrance corporelle. C'est la concentration audio-visuelle qui les aide à se détacher de leurs corps et lorsqu'elle s'affaiblit, elle les empêche de retrouver leur univers spirituel. Face à la torture, certains d'entre eux se dégagent "de leur corps par un effort de concentration très puissant, ce qui leur permettait de ne plus souffrir" (2001, p. 10). C'est le cas de Salim, persuadé que la maîtrise de ses pensées et de ses émotions lui permet de se 
protéger et de bien réagir face à tout danger. Il avait la capacité de "maîtriser [son] corps dans le grand froid, dans la chaleur étouffante, dans les crises de rhumatisme" (p. 129). Nous l'avons vu changer sans cesse la position de son corps afin d'éviter toute souffrance corporelle; il avait de même l'habitude de penser au printemps et à la lumière en vue de ne pas sentir l'odeur infecte de merde. Salim résume sa méthode à travers ces mots: "Être là sans être là. Fermer ses sens, les diriger ailleurs" (p. 12).

Concret et tangible, le corps renferme l'âme abstraite; les deux constituent ensemble "un système complexe dans lequel notre corps influence nos pensées et où nos pensées influencent notre corps" (Regard, 2007, p. 18). Lorsqu'un homme est fâché, sa colère est accompagnée de crispations physiques; lorsqu'il est menacé, son corps devient plein d'énergie afin d'affronter cette menace et vice versa. L'homme qui tombe amoureux, devient solide et en pleine forme. Cependant, chacun a sa propre façon de ressentir une émotion, influencé par son système nerveux et hormonal. A ce propos, Regard présente la technique de désensibilisation, visant à "réaliser des séries de mouvements oculaires horizontaux en même temps qu'on pense à une émotion désagréable" (p. 183). Cette technique aide l'homme à atténuer sa charge émotionnelle et à sa délivrer de tout ce qui est perturbant: stress, anxiété, jalousie ... etc. Avec cette technique, l'homme peut désensibiliser ses émotions, il ressemble au dentiste qui dévitalise le nerf d'une dent. Cette technique se passe à travers deux étapes: Identifier l'émotion qui est à l'origine de la douleur et penser en même temps à d'autres choses jusqu'à ce que cette émotion disparaisse ou soit inactive. D'après Regard, "lorsqu'une émotion est neutralisée, elle ne produit plus aucun effet. Elle n'a plus aucun intérêt et ne peut plus nous faire souffrir" (p. 193). Les détenus de Tazmamart ont utilisé cette technique face au froid qui

(Prison et liberté d'après ...) Dr. Ayman El Ghandour 
a gelé leur sang; ils ont essayé de s'occuper d'autres choses. A titre d'exemple, le personnage de Karim était soucieux de bien déterminer le temps en vue de ne pas prendre conscience de sa douleur. Aussi Salim a-t-il tenté de se délivrer de la captivité de son corps pour atteindre l'esprit en maîtrisant le rythme de sa respiration. Après avoir réussi à se calmer, il nous annonce ce qu'il a fait: "J'ai nettoyé ma mémoire. Je l'ai débarrassée des souvenirs trop douloureux à évoquer" (2001, p. 135).

A travers le personnage de Salim, Ben Jelloun ne s'est pas contenté de présenter la pensée comme un moyen d'arrachement au monde carcéral, mais il a introduit un écart religieux. Pour oublier son corps, le héros s'est focalisé sur "une pierre sacrée située à des milliers de kilomètres, à des siècles de [sa] cellule" (2001, p. 62). Ceci exige que le héros, emprisonné à Tazmamart, aille à la Mecque afin de toucher la pierre noire. Comme ce voyage était impossible sur le plan matériel, Salim a dépendu sur la charge modale qui "ouvre un espace sémiotique imaginaire où le discours passionnel peut se déployer" (Greimas et Fontanille, 1991, p. 59). Cet espace imaginaire lui a permis de contrôler sa pensée et de bien garder ses sens et son désir de survie. Donc son voyage est adressé vers l'intérieur.

C'est la prière qui a aidé Salim à s'apaiser et à dépasser sa douleur. Il y a trouvé son issue d'un monde physique perturbant. Grâce à elle; il "se détache petit à petit de son corps, s'en éloignant pour ne pas être l'esclave de ses souffrances, de ses appétits et de ses délires" (2001, p. 189). Il a commencé son itinéraire soufi, se consacrant à Allah, l'aimant sans crainte. Le protagoniste nous affirme qu'il est arrivé "grâce aux prières et à la récitation de poèmes soufis, à atténuer l'intensité de la douleur" (p. 190) Rappelons ici que le soufisme "est le 
renoncement à tous plaisirs égoïstes" (Meyerovitch, 2005, p. 82). En l'adoptant, Salim a atteint une contemplation véritable et une annihilation entière où il était détaché de tout ce qui l'entourait. Le soufisme lui a permis de dépasser l'obscurité de la cellule et d'apercevoir la lumière spirituelle dont la recherche "guide le pas de plusieurs protagonistes des récits benjellouniens" (Ionicescu, 2009, p. 139).

Malika et Tahar ont abordé le conte et le rêve, deux éléments absents dans le roman de Latifa Al-Zayyat. Loin d'être gratuits, ils ont aidé les détenus de Tazmamart à résister aux conditions pénibles, garder leur équilibre intérieur jusqu'au bout. D'une part, le rêve a révélé la pensée et l'intériorité des détenus; il leur a permis d'obtenir momentanément leur liberté, de toucher l'avenir ou de remonter au passé, "de faire un retour au monde extérieur, inaccessible aux condamnés à la mort lente" (Ndagijamana, 2007, p. 234); d'autre part, tout conte inséré dans un récit, a une double fonction: divertir les lecteurs et leur donner des leçons de morale.

De sa part, Malika a montré l'importance des rêves qui l'avaient placée dans un monde fictionnel. Elle déclare: "la nuit était propice aux songes qui nous aidaient à nous échapper, à lire dans notre avenir" (1999, p. 239). Elle et ses frères se sont livrés aux rêveries. Lorsqu'ils pensaient avoir quarante-huit heures de liberté, chacun d'entre eux a rêvé de réaliser un souhait: Mimi a voulu voler des fèves; Raouf avait le désir de rencontrer des femmes, Malika, elle-même, a souhaité aller à une librairie et tomber amoureuse avec un homme. De son côté, Salim a constaté que ses nuits étaient pleines de rêves. Il a affirmé qu'il était "le seul à rêver des trois prophètes" (2001, p. 155); il s'agit de Moïse, Jésus et Mohamed qui représentent pour lui un appui divin. Ils

(Prison et liberté d'après ...) Dr. Ayman El Ghandour 
l'ont invité à la patience afin de fuir sa situation pénible. Les rêves n'étaient pas bornés sur Salim; les autres détenus avaient l'habitude de rêver, lors de l'approche de la mort. Mentionnons à titre d'exemple le rêve d'Abbass où "une main blanche descend $d u$ ciel, passe entre les grilles et s'empare d'un pigeon" (p. 157).

En ce qui concerne le conte, le personnage de Malika ressemble à Schéhérazade. Grâce à ses contes, cette dernière a pu sauver tant de femmes, destinées à la mort par Chahrayar. Malika a bien joué le même rôle, essayant "d'omettre le temps ennemi en prison et de solliciter la compassion d'un roi tortionnaire" (Farhoud, 2008, p. 165). Conteuse habile et intarissable, elle a créé des mondes d'imagination, marqués par la présence de la liberté. Elle considère ainsi le mot comme une arme face à la mort, à la folie, au désespoir et à la vie carcérale. C'est ce qui l'a poussée et sa famille à inventer des mots incompréhensibles. Elle dit: "Nous parlions par allusions, nous utilisons un langage codé, que nous seuls comprenions" (1999, p. 229). Rappelons ici ce qu'a écrit Ahmed Marzouki, un des détenus réels de Tazmamart, dans son livre intitulé Tazmamart cellule 10: "La nécessité de disposer d'un langage compréhensible de nous seuls s'est imposée" (2001, p. 120); il s'agit du Tazmamarti dont nous citons les mots suivants:

\begin{tabular}{|c|c|}
\hline Tazmamarti & Sens en français \\
\hline Alfa & Algérie \\
Corbeau & Avocat \\
Écho & Égypte \\
Bikenboer & Allemagne \\
Pepsi cola & Ligue arabe \\
Sippo & Soldat \\
\hline
\end{tabular}

(Prison et liberté d'après ...) Dr. Ayman El Ghandour 
Quant à Ben Jelloun, il est conscient du rôle salvateur de la parole. Ceci est évident avec Salim, le narrateur homodiégétique de son récit, qui s'avère un excellent conteur; sa présence est cruciale pour ses compagnons parce que ses contes leur présentent momentanément un soulagement de leur douleur. Ceux-ci ont bien écouté le conte d'un homme à la fois riche et avare qui "épousa plusieurs femmes, mais aucune ne réussit à lui donner un enfant" (2001, p. 88). Curieux et interactifs, ils ont demandé à Salim d'éclaircir les points ambigus. Il leur a de même raconté des films et d'autres genres littéraires telle la poésie, particulièrement celle de Paul Éluard, basée sur la lumière et la liberté. Ses contes ont contribué à approfondir le genre narratif dans le roman qui "consiste avant tout en une histoire racontée que l'on appellera tour à tour, et suivant les modes littéraires: narration, récit ou fiction" (Valette, 1993, p. 19); ils ont aussi atténué la douleur des détenus et les ont fait voyager dans un monde imaginaire, distingué par la paix et la sérénité.

A la fin de notre étude, nous avons constaté que Latifa AlZayyat Malika Oufkir et Tahar Ben Jelloun avaient condamné les régimes despotiques soit en Égypte soit au Maroc. Ils ont dénoncé les mesures répressives qu'avaient prises le président Sadate et le roi Hassan II pour éliminer leurs opposants en les enfermant dans des prisons inhumaines: Kanater, Bir Jdid et Tazmamart. Ce dernier qui est le plus terrible, symbolise la dictature sous toutes ses formes. Chacun d'entre eux a bien présenté le contexte politique et historique de son pays. Grâce à eux, nous avons vu de près la défaite de 67, le triomphe d'octobre, les accords de Camp David, l'encouragement de Sadate pour les islamistes, son agressivité contre les communistes, la mort de Mohamed V, la venue de Hassan II, le putsch de Skhirat et les années de plomb.

(Prison et liberté d'après ...) Dr. Ayman El Ghandour 
Les trois romanciers ont exprimé en détails la vie carcérale, la souffrance des détenus, leur douleur physique et psychique.

En ce qui concerne la technique romanesque, nous avons remarqué une différence entre les ouvrages de Latifa et Malika d'une part et celui de Ben Jelloun d'autre part. Les deux premières ont adopté le récit autobiographique où la narratrice, l'héroïne et l'écrivaine sont identiques et portent le même nom. Leur narration homodiégetique a exigé la prédominance de "je", ce pronom qui rend le récit crédible et exprime fidèlement les sentiments du narrateur. Mais le "je" narratif dans La prisonnière représente plusieurs voix parce que Malika raconte à la fois son emprisonnement et celui de sa famille, alors que Ben Jelloun a écrit un récit fictionnel dédié à Aziz Binebine, mêlant la réalité à la fiction. Cependant, il a utilisé le pronom "je" pour prendre une place dans l'événement qu'il n'a jamais vécu. Il a donné l'acte de narrer à Salim qui a intégré ses paroles et celles d'autres détenus. Bien que les trois romans abordent la vie carcérale, la prison est à la base de Cette aveuglante absence de lumière, elle est partout, presque omniprésente dans chaque page; alors que Latifa et Malika y ont consacré une partie dans leurs romans; l'une a fait émerger à la fois son évolution personnelle, les événements politiques et la société qui l'entourait, consacrant à la fin de son ouvrage, une partie à la perquisition. L'autre a abordé son enfance, son éducation au palais royal, arrivant au coup d'État en 1972 qui a mis fin à son père et a conduit la famille Oufkir à la prison. Comme les deux écrivains marocains ont respecté l'ordre chronologique, leurs romans sont linéaires, contrairement à Perquisition d'Al-Zayyat, ouvrage composé de fragments dispersés. 
Les trois romanciers ont bien décrit la violence politique et légitime qu'avait adoptée l'État policier face à ses opposants. Les personnages sont bien esquissés et incarnent les victimes d'un régime despotique; ils ont pu dompter leurs pensées et leurs émotions afin de résister aux conditions inhumaines pendant leur emprisonnement. Aussi les contes et les rêves les ont-ils aidés à résister jusqu'au bout. Leur survie est significative parce qu'elle affirme l'aptitude du bien à vaincre le mal. Ici réside le message essentiel de l'écriture carcérale qui vise à dénoncer la violence sous tous ses aspects, à commémorer les détenus politiques en les plaçant au cœur des textes et par conséquent à la mémoire collective. 


\section{Bibliographie}

\section{1- Corpus étudié :}

- Ben Jelloun, T. (2001). Cette aveuglante absence de lumière. Paris: Éditions Du Seuil.

- Oufkir, M. (1999). La prisonnière. Paris: Éditions Grasset et Fasquelle.

- Zayyat, L. (1992/1996). Perquisition. (Traduit de l'arabe par Richard Jacquemond). Paris: Actes Sud.

\section{2- Ouvrages généraux :}

- Bazié, I. et Ferrer, C. (2015). Écritures de la réclusion. Québec: Presses de l'Université du Québec.

- Coquio, C. (2015). La littérature en suspens. Paris: L'Arachnéen.

- Crettiez, X. (2008). Les formes de la violence. Paris: Éditions La découverte.

- Dadoun, R. (1993). La violence: Essai sur l'«homo violens». Paris: Hatier.

- Desjardins, T. (1981). Sadate pharaon d'Égypte. Paris: Éditions Marcel Valtat.

- Dulong, R. (1998). Le témoin oculaire: Les conditions sociales de l'attestation personnelle. (Coll. Recherches d'histoire et de sciences sociales). 79. Paris: EHESS.

- Ekotto, F. (2001). L'écriture carcérale et le discours juridique chez Jean Genet. (Coll. Critiques littéraires). Paris: L'Harmattan.

- Evrard, F. (2006). Jeux autobiographiques. Paris: Ellipses Éditions Marketing S.A.

- Foucault, M. (1975). Surveiller et punir: Naissance de la prison. Paris: Gallimard.

- Genette, G. (1991). Fiction et diction. Paris: Éditions Du Seuil.

- Greimas, A. et Fontanille, J. (1991). Sémiotique des passions: Des états de choses aux états d'âmes. Paris: Éditions Du Seuil.

- Gusdorf, G. Les écritures du moi: Autobiographie: Lignes de vie. Paris: Éditions Odile Jacob.

- Le Breton, D. (2018). La sociologie du corps. (Que sais-je). Paris: PUF.

(Prison et liberté d'après ...) Dr. Ayman El Ghandour 
- Lejeune, P. (1996). Le pacte autobiographique (Coll. Points). Paris: Éditions Du Seuil.

- Marzouki, A. (2001) Tazmamart cellule 10. Paris: Gallimard.

- Meyerovitch, E. (2005). Rûmî et le soufisme. Paris: Éditions Du Seuil. (Coll. Sagesse).

- Perrault, G. (1990). Notre ami le roi. Paris: Gallimard.

- Rachid, A. (2020). Écriture de soi et révolution. Dans Mobarak, S. (dir.), Amina Rachid ou La traversée vers l'autre. 102-110. Casablanca: Centre culturel du livre.

- Regard, J. (2007). Les émotions. Paris: Eyrolles.

- Ricoeur, P. (2000). La mémoire, l'histoire, l'oubli. Paris: Éditions Du Seuil.

- Solé, R. (2013). Sadate. Paris: Perrin.

- Souiller, D. et Troubetzkoy, W. (1999). Littérature comparée. Paris: PUF.

- Valette, B. (1993). Esthétique du roman moderne. Paris: Nathan.

\section{3- Revues et périodiques :}

- Attafi, A. (2012). Le soufisme dans "Cette aveuglante absence de lumière" de Tahar Ben Jelloun. Dans Nouvelles études francophones. 27 (1), 194-205. Repéré à https//:www.jstor.org/stable/24245386.

- Balta, P. (1999). Hassan II du féodal au (presque) libéral. Dans Confluences méditerranée. (31), 25-33. Repéré à https://www.editionsharmattan.fr/index.asp? navig $=$ catalogue $\&$ obj $=$ article $\&$ no $=3046$

- Éditions Esprit. (1956). Tribunaux et bagnes d'Égypte. 237 (4), 610623. Repéré à https//:www.jstor.org/stable/24254238.

- Fähndrich, H. (1999). Qu'écrit-on dans les autobiographies arabes modernes? : Les exemples de Abd ar-Rahmn Munf, Latfa az-Zayyt et Hlid Ziyda. Dans Asiatische Studien : Zeitschrift der Schweizerischen Asiengesellschaft (Études asiatiques: revue de la société Suisse-Asie). (53), 75-85. Repéré à https://www.e-periodica.ch/cntmng?pid=ast002:1999:53::1073

- Khalaf, N. (2015). Repenser le nassérisme (1952-1970) (Autour d'Anouar Abdel-Malek (1924-2012). Dans Revue française d'Histoire des idées politiques, (42), 47-89. Repéré à https//:www.jstor.org/stable/24610763.

(Prison et liberté d'après ...) Dr. Ayman El Ghandour 
- Mésavage, R. (2004). Espace carcéral et imaginaire dans les écrits de Marzouki, d'Oufkir et de Serhane. Dans Nouvelles études $\begin{array}{lllll}\text { francophones. } & 19 & (2), & 183-196 . & \text { Repéré à }\end{array}$ https//:www.jstor.org/stable/25701861.

- Monciaud, D. (2008). Une trajectoire féminine dans la gauche égyptienne: dimensions et enjeux des engagements de Soraya Adham (1926-2008). Dans Cahiers d'histoire: Revue d'histoire critique. (105106), 235-285. Repéré à https://journals. openedition.org/chrhc/550

- Saraya, M. (2020). Le récit de prison comme témoignage sur l'espace hostile: Barraux de Mohammed Al Bossaty, Le baiser de la femmearaignée de Manuel Puig et Le dernier jour d'un condamné de Victor Hugo. Dans Bulletin of the Faculty of Arts, Université du Caire. (80), 97-130.

- Solé, R. (2014). L'Égypte d'une révolution à l'autre. Dans Bulletin de la Sabix. (54), 53-57. Repéré à http://sabix.revues.org/1121.

-Tadié, A. (1996). L'univers des romancières égyptiennes: thèmes et formes dans la littérature féminine. Dans Peuples méditerranéens, (77), $47-94$.

\section{4- Thèses universitaires:}

-Farhoud, S. (2009). Interventions autobiographiques au Maghreb: L'écriture comme moment de transmission des voix de femmes. Thèse de doctorat. Université de Montréal. Repéré à https://0811jpyi3-1106y-https-www-proquest-com.mplbci.ekb.

eg/docview/230901704/D841293F79C8488BPQ/7? accountid=178282.

- Ionicescu, A. (2009). Lectures de sable: Les récits de Tahar Ben Jelloun. Thèse de doctorat. Université de Rennes 2. Repéré à https://core.ac.uk/download/pdf/ 54025958.pdf

- kadji, D. (2020). Comparatisme du conte francophone: Poétiques de la réécriture et enjeux transtextuels chez Tahar Ben Jelloun et Amélie Nothomb. Thèse de doctorat. Université de L'Albama. Tuscaloosa. Repéré à https://0811jw1iv-1105-y-https-www-proquestcom.mplbci.ekb.eg/docview/2447559722/F14AF98D4C764588PQ/1? accountid $=178282$

- Ndagijimana, E. (2007). La mémoire de la violence dans le roman africain contemporain. Thèse de doctorat. Université de Montréal.

(Prison et liberté d'après ...) Dr. Ayman El Ghandour 
Repéré à https://0811jp7fw-1104-y-https-www-proquestcom.mplbci.ekb.eg/docview/304743533/

E69101533B8A4CA3PQ/1?accountid=178282

- Wand, J. (1993). Tahar Ben Jelloun: Auteur de la différence. Thèse de doctorat. Université de San Jose State. California. Repéré à https://0811jp7c0-1104-y-https-www-proquestcom.mplbci.ekb.eg/docview/304100810/33129BA21BF641A5PQ/1? accountid $=178282$

\section{5- Sites Web:}

- Ayad, C. (1996). Latifa femme du siècle. Repéré le 5/6/2021 à https://www.liberation.fr/livres/1996/10/17/latifa-femme-dusiecletraduction-de-l-autobiographie-inachevee-d-une-intellectuelle-degauche-et-nat_186674/

- Ben Jelloun, T. (S.D.). Repéré le 5/5/2021 à https://citationcelebre.leparisien.fr /citation/la-liberte-n-est-rien-si

- Hallaq, B. (1996). Une femme égyptienne. Dans Le monde diplomatique. Repéré le 15/3/2021 à https://www.mondediplomatique.fr/1996/12/HALLAQ/5993

- Haubruge, P. (2001). Ben Jelloun, aveugle? Polémique autour de son nouveau roman, sur le bagne marocain de Tazmamart. Dans Le Soir. Repéré le 23/2/2021 à https://www.lesoir.be/art/ben-jelloun-aveuglepolemique-autour-de-son-nouveau-rom_t-20010124-Z0K5QE.html

- Le Vaillant, L. (1999). Portrait, Malika Oufkir. Repéré le 20/5/2021 à https://www.liberation.fr/portrait/1999/02/22/malika-oufkir-45-ans-enexil-apres-vingt-ans-de-detention-la-fille-du-general-qui-voulutchasser-has_265534/

- Maroc, Constitution du 7 décembre 1962. Repéré le 30/3/2021 à https://mjp.univ-perp.fr/constit/ma1962.htm

- Trierweiler, V. (2011). Tahar Ben Jelloun et Benjamin Stora : La saison de la liberté. Dans Paris match. Repéré le 23/2/2021 à https://www.parismatch.com/ Culture/Livres/Tahar-Ben-Jelloun-etBenjamin-Stora-La-saison-de-la-liberte147936?fbclid=IwAR3jhDPdkQ5PsB5Qe4Diz8IFNjszzmt9p6Kn6ITI61 styxO_M1P-HjYkJms

(Prison et liberté d'après ...) Dr. Ayman El Ghandour 
- Trutt, J.-C. (2019). Trois féministes égyptiennes. Repéré le 7/3/2021 à https://jean-claude-trutt.com/bloc-notes/trois-feministes-egyptiennes

\section{6-Vidéos sur You tube:}

- France 24. (2019). L'invité du jour: Tahar Ben Jelloun. [Vidéo en ligne ]. Accès https://www.youtube.com/watch?v=aoG5h0Dl-As

- L'établissement de détention de Montréal. (1999). Malika Oufkir chez les Souverains extrait 2. [Vidéo en ligne ]. Accès https://www.youtube.com/watch?v= TM5CTBu5YMc 
السجن والحرية لاى لطيفة الزيات ومليكة أوفقير والطاهر بن جلون

\section{ملخص}

تركز هذه الدراسة على أدب السجون من خلال روايات "حملة تفنيش" للطبفة الزيات ،

و "السجينة" لمليكة أوفقير و "تلك العتمة|لباهرة" للطاهر بن جلون. قدمت لطيفة ومليكة قصتين عن سيرتهما الذاتية بينما مزج الطاهر بن جلون الواقع بالخيال في قصته المهداة لعزيز بنبن. لقد وصف الروائيون الثناثة بشكل جيد العنف السياسي الذي تمارسه الدولة ضد معارضيها ، مستتكرين النظام الاستبدادي في بلادهم. وألقوا الضوء على الإجراءات القمعية ، معبرين عن معاناة المعتقلين في السجون اللا إنسانية: بير جديد ، القناطر ونازمامارت. و تمكن السجناء السياسيون في الأعمال الثلاثة من ترويض عواطفهم من أجل مقاومة الظروف القاسية أثناء احتجازهم. و يبدو هذا واضحًا من خلال شخصية سليم الذي كرس نفسه للتصوف.

الكلمات المفتاحية: أدب السجون ، السجن ، الحرية ، تازمامارت ، السيرة الأتية. 